\title{
Strategic hospital partnerships: improved access to care and increased epilepsy surgical volume
}

\author{
Sumeet Vadera, MD, Alvin Y. Chan, BS, Lilit Mnatsankanyan, MD, Mona Sazgar, MD, \\ Indranil Sen-Gupta, MD, Jack Lin, MD, and Frank P. K. Hsu, MD, PhD \\ Comprehensive Epilepsy Surgery Center, University of California, Irvine, California
}

\begin{abstract}
OBJECTIVE Surgical treatment of patients with medically refractory focal epilepsy is underutilized. Patients may lack access to surgically proficient centers. The University of California, Irvine (UCl) entered strategic partnerships with 2 epilepsy centers with limited surgical capabilities. A formal memorandum of understanding (MOU) was created to provide epilepsy surgery to patients from these centers.
\end{abstract}

METHODS The authors analyzed UCl surgical and financial data associated with patients undergoing epilepsy surgery between September 2012 and June 2016, before and after institution of the MOU. Variables collected included the length of stay, patient age, seizure semiology, use of invasive monitoring, and site of surgery as well as the monthly number of single-surgery cases, complex cases (i.e., staged surgeries), and overall number of surgery cases.

RESULTS Over the 46 months of the study, a total of 104 patients underwent a total of 200 operations; 71 operations were performed in 39 patients during the pre-MOU period (28 months) and 129 operations were performed in 200 patients during the post-MOU period (18 months). There was a significant difference in the use of invasive monitoring, the site of surgery, the final therapy, and the type of insurance. The number of single-surgery cases, complex-surgery cases, and the overall number of cases increased significantly.

CONCLUSIONS Partnerships with outside epilepsy centers are a means to increase access to surgical care. These partnerships are likely reproducible, can be mutually beneficial to all centers involved, and ultimately improve patient access to care.

https://thejns.org/doi/abs/10.3171/2018.1.FOCUS17683

KEYWORDS NAEC; epilepsy center; patient care strategies

$\mathrm{T}$ HE literature suggests that surgery improves outcomes for patients with medically refractory epilepsy, and that surgical treatment can be potentially curative. ${ }^{11,12,18}$ The American Academy of Neurology recommends that appropriate patients should be referred to an epilepsy surgery center for evaluation following failure of an appropriate trial of medical treatment (i.e., persistent seizures despite a trial of at least 2 antiepileptic drugs [AEDs]). ${ }^{4}$ Despite this recommendation, epilepsy surgery is underutilized in the United States. The number of operations performed for pediatric patients increased from 2000 to $2009,{ }^{14}$ but the number of overall surgeries remains stagnant. ${ }^{5,16} \mathrm{~A}$ lack of access to surgically proficient medical centers and poor referral rates from nonsurgical epilepsy centers are possible causes. Studies have shown that patients with intractable epilepsy were referred for surgery later in the disease course than recommended, which could have been due to a lack of awareness of current surgical referral guidelines. ${ }^{8,15}$

We developed strategic partnerships with 2 nearby epilepsy centers to provide access to surgery for patients with medically refractory epilepsy. Preoperative workups were performed by the referring neurologist, and cases were presented via video-teleconference during a dedicated epilepsy surgery conference. We sought to examine how these partnerships affected patient access to surgery and what impact this had upon institutional profitability as well as to provide a model by which other institutions may increase epilepsy surgical volume.

\section{Methods}

\section{Strategic Partnership}

The National Association of Epilepsy Centers (NAEC)

ABBREVIATIONS AED = antiepileptic drug; MEG = magnetoencephalography; $M O U$ = memorandum of understanding; NAEC = National Association of Epilepsy Centers; $\mathrm{PET}$ = positron emission tomography; RNS = responsive neurostimulation; SDG = subdural grid electrode; SEEG = stereo-encephalography; UCI = University of California, Irvine; $v E E G$ = video electroencephalography; VNS = vagus nerve stimulation.

SUBMITTED November 8, 2017. ACCEPTED January 18, 2018.

INCLUDE WHEN CITING DOI: 10.3171/2018.1.FOCUS17683. 
TABLE 1. Formally agreed-upon terms of the memorandum of understanding

1. All preoperative surgical workup would be performed at referring center (e.g., vEEG, 3-T MRI, PET, MEG, neuropsychiatry evaluation, etc.); no preoperative studies would be repeated at $\mathrm{UCl}$ except for presurgical planning purposes (e.g., navigation imaging studies).

2. The referring epileptologist would present the case either via video-teleconference or in person, and would present all workup to date to entire UCI epilepsy team.

3. The outcome of the conference would be either surgical referral or further workup (performed at the primary referring site).

4. If referred for surgery, the patient would be seen by a UCI neurosurgeon (e.g., S.V. or F.P.K.H.), and informed consent for treatment would be obtained. The patient would also be evaluated by a UCl epileptologist for perioperative monitoring.

5. After surgery, the patient would be followed by a UCl epileptologist in house and for 6 weeks postoperatively. The neurosurgeon would follow in house and per individual surgeon protocol.

6. The patient would be transitioned back to the care of the primary epileptologist 6 weeks postoperatively.

designated the University of California, Irvine (UCI) the only adult level 4 epilepsy center with surgical capabilities in Orange County, California, one of the most populous counties in the United States. We developed partnerships with 2 outside epilepsy centers, Loma Linda and Kaiser Sunset, to surgically treat patients who were deemed surgical candidates. Both outside centers were performing fewer than 10 noncomplex surgeries per year (i.e., no complex or multiple-stage surgeries). A memorandum of understanding (MOU) was made with both partners based on formally agreed-upon terms (Table 1). This allowed our physicians to treat the patients from the partner centers and accept their insurance coverage. An official administrative meeting was held between us and each partner to ensure complete agreement upon the terms.

All patients considered for surgery from both institutions were referred-not only a subset-because the referring centers had a backlog of surgical candidates (i.e., epilepsy patients whose seizures were not adequately controlled with AEDs). The referring epileptologist had the ultimate decision in terms of prolonging medical treatment or referring for surgical intervention. The referring epileptologist then completed the entire presurgical workup, which included video electroencephalography (vEEG), imaging studies (e.g., 3-T MRI, positron emission tomography [PET], single-photo emission computed tomography [SPECT], magnetoencephalography [MEG], etc.), and medication management at the referring center, which maintained continuity of care and revenue for the outside center. Patients deemed surgical candidates were seen by UCI epilepsy surgeons (S.V. or F.P.K.H.) and underwent preoperative evaluation. During the surgical and perioperative periods, UCI epileptologists monitored the patients, adjusted medications, and assessed all encephalographic data. Patients were transitioned back to the referring center and epileptologist 6 weeks after surgery and continued to be followed by the epilepsy surgeon per routine.

\section{Epilepsy Conference}

Due to the geographical distance, a live video stream was developed to facilitate participation of clinicians from each center and UCI in the epilepsy surgical conferences. We utilized standard teleconference equipment freely available at our institution and the partner institutions, as well as online conference tools. The information technology department at our institution was in charge of main- taining the equipment. No additional full-time employees were required. There was an epilepsy teleconference every 2 weeks with each referral site, and at each teleconference 3-5 cases were presented. UCI epileptologists, neurosurgeons, neuroradiologists, and neuropsychiatrists attended each conference, as well as the referring partner epileptologist who presented his or her patients. All imaging studies, vEEG data, neuropsychological testing data, and clinical findings were discussed. A treatment consensus was reached among all members of the group and tailored to the patient's unique clinical presentation. Surgical procedures included resective surgery, implantation of depth electrodes for stereo-encephalography (SEEG), implantation of subdural grid electrodes (SDGs) for SDGbased EEG, implantation of responsive neurostimulation (RNS) systems, implantation of vagus nerve stimulation (VNS) devices, laser ablation, and palliative surgeries such as corpus callosotomy. If the patient underwent electrode implantation, the UCI epileptologist caring for that patient presented the EEG findings to the teleconference group in a follow-up epilepsy conference prior to any treatment performed (e.g., RNS, VNS), which allowed the referring epileptologist to be a member of the decisionmaking team.

\section{Data Collection}

The study was conducted following STROBE (Strengthening the Reporting of Observational Studies in Epidemiology) guidelines. We retrospectively collected and analyzed data from September 2012 through June 2016. All patients undergoing epilepsy surgery during this time frame were eligible for data collection. Referrals started in January 2015; thus the pre-MOU period was 28 months long and the post-MOU period 18 months. Data for the following variables were collected: 1 ) number of surgeries per patient, 2) whether the case was referred or primary to UCI, 3) the admission date, 4) length of stay in days, 5) seizure semiology (e.g., complex vs partial), 6) anatomical site of surgery, 7) whether invasive monitoring was used, 8) the final surgical therapy provided (e.g., neurostimulation, resection), and 9) complications. Financial data for the following variables were collected from the UCI financial department in US dollars: 1) total revenue, 2) contribution margins, 3) profit margins, and 4) total costs. The insurance provider was also noted. We combined continuous data (e.g., financial data) for separate admissions in 
TABLE 2. Summary descriptive data for the 18-month period after partnership development

\begin{tabular}{|c|c|c|c|c|}
\hline \multirow[b]{2}{*}{ Variable } & \multicolumn{4}{|c|}{ Post-MOU } \\
\hline & $\mathrm{UCI}(\mathrm{n}=36)$ & Partner 1 (Kaiser; $n=12$ ) & Partner 2 (Loma Linda; $\mathrm{n}=17$ ) & $p$ Value \\
\hline Patient age (yrs), mean & $36.0 \pm 2.2$ & $37.5 \pm 4.3$ & $38.3 \pm 3.6$ & 0.809 \\
\hline LOS (days), mean & $6.7 \pm 0.8$ & $8.6 \pm 2.6$ & $9.4 \pm 1.5$ & 0.218 \\
\hline Seizure semiology & & & & 0.170 \\
\hline Complex partial & $33.3 \%$ & $58.3 \%$ & $50.0 \%$ & \\
\hline Simple partial & $44.4 \%$ & $0.0 \%$ & $5.6 \%$ & \\
\hline Generalized & $8.3 \%$ & $16.7 \%$ & $27.8 \%$ & \\
\hline Unspecified & $13.9 \%$ & $25 \%$ & $16.7 \%$ & \\
\hline Invasive monitoring & & & & 0.024 \\
\hline Yes & $63.9 \%$ & $41.7 \%$ & $88.9 \%$ & \\
\hline No & $36.1 \%$ & $58.3 \%$ & $11.1 \%$ & \\
\hline Surgery site & & & & 0.007 \\
\hline Temporal & $52.8 \%$ & $100.0 \%$ & $61.1 \%$ & \\
\hline Extratemporal & $36.1 \%$ & $0.0 \%$ & $11.1 \%$ & \\
\hline Other & $11.1 \%$ & $0.0 \%$ & $28.8 \%$ & \\
\hline Surgical therapy & & & & 0.021 \\
\hline Resection & $47.2 \%$ & $91.7 \%$ & $83.3 \%$ & \\
\hline Neurostimulation & $36.1 \%$ & $8.3 \%$ & $11.1 \%$ & \\
\hline Other & $16.7 \%$ & $0.0 \%$ & $5.6 \%$ & \\
\hline Insurance & & & & $<0.001$ \\
\hline Commercial/private & $22.3 \%$ & $75.0 \%$ & $5.6 \%$ & \\
\hline Government & $69.4 \%$ & $25.0 \%$ & $88.9 \%$ & \\
\hline $\mathrm{HMO}$ & $8.3 \%$ & $0.0 \%$ & $5.5 \%$ & \\
\hline Electrode type & & & & 0.166 \\
\hline SEEG (depth) & $22.2 \%$ & $25.0 \%$ & $41.2 \%$ & \\
\hline SDG & $41.7 \%$ & $16.7 \%$ & $41.2 \%$ & \\
\hline None & $36.1 \%$ & $58.3 \%$ & $17.6 \%$ & \\
\hline
\end{tabular}

$\mathrm{HMO}=$ health maintenance organization; LOS = length of stay.

Data in the $\mathrm{UCl}$ column are from cases that were primary to $\mathrm{UCl}$. Data in the partner columns are for cases referred from those institutions. Data are percentages of cases unless otherwise indicated. Means and standard deviations are provided for continuous data. Boldface type indicates statistical significance.

cases involving staged procedures requiring multiple admissions. The admission date and age were taken from the first admission. The study was approved by the UIC institutional review board.

\section{Statistical Analysis}

Differences in means were investigated via unpaired t-tests or analyses of variance (ANOVAs) for continuous data and chi-square tests for categorical. Fisher transformation tests were used to test for correlation between variables. Wizard Pro (v. 1.8.17) was used to analyze the data and create the figures. Statistical biases were considered, and none were identified.

\section{Results}

A total of 104 patients were treated during the overall study period (46 months), with 39 patients being treated during the pre-MOU period (28 months) and 65 during the post-MOU period (18 months). These 104 patients underwent a total of 200 surgeries -71 in the pre-MOU period and 129 in the post-MOU period. The descriptive statis- tics for the post-MOU period, stratified by institution, are summarized in Table 2. Comparison of data for primary UCI cases and referrals from the 2 partner institutions showed no significant difference with respect to patient age, length of stay, or seizure semiology. There was a significant difference in use of invasive monitoring, anatomical site of surgery, surgical therapy, and type of insurance. The data for the average number of cases per month for both the prepartnership and postpartnership periods are summarized in Table 3 . There was a significant difference between the pre- and postpartnership data for the number of single-surgery cases per month (i.e., cases in which patients underwent only a single-stage procedure), number of complex surgery cases per month (i.e., cases in which patients underwent surgery in 2 or more stages), and number of overall cases per month.

Table 4 shows the overall counts for cases in which patients underwent SDG electrode implantation and SEEG depth electrode implantation as well as the final therapy after ictal-onset localization. There was a significant positive correlation between length of stay and revenue ( $\mathrm{r}=$ $0.658, \mathrm{p}<0.001)$. The complications and rates were in line 
TABLE 3. Comparison of mean monthly numbers of cases and financial data for the pre- and post-MOU periods

\begin{tabular}{|c|c|c|c|}
\hline \multirow[b]{2}{*}{ Variable } & \multicolumn{2}{|c|}{ Mean $(95 \% \mathrm{Cl})$} & \multirow{2}{*}{$\begin{array}{c}p \\
\text { Value }\end{array}$} \\
\hline & Pre-MOU & Post-MOU & \\
\hline 1 surgery, no. of cases per month & $0.43(0.11-0.75)$ & $1.28(0.52-2.04)$ & 0.02 \\
\hline $2+$ surgeries, no. of cases per month & $0.96(0.62-1.30)$ & $2.39(1.85-2.93)$ & $<0.01$ \\
\hline Overall no. of cases per month & $1.39(0.98-1.81)$ & $3.67(2.83-4.50)$ & $<0.01$ \\
\hline Total monthly charge & $\$ 597,007.01(\$ 321,606.31-\$ 872,407.71)$ & $\$ 1,007,488.65(\$ 767,458.17-\$ 1,247,519.13)$ & 0.04 \\
\hline Total monthly revenue & $\$ 130,984.12(\$ 58,264.53-\$ 203,703.71)$ & $\$ 214,059.50(\$ 137,394.45-\$ 290,724.55)$ & 0.12 \\
\hline Total monthly contribution margins & $\$ 58,476.77(\$ 9,080.65-\$ 107,872.98)$ & $\$ 76,689.85(\$ 23,145.28-\$ 130,234.42)$ & 0.62 \\
\hline Total monthly profit margins & $\$ 15,662.63(-\$ 27,850.93$ to $\$ 59,176.19)$ & $-\$ 12,463.82(-\$ 58,259.86-\$ 33,332.22)$ & 0.38 \\
\hline Total monthly cost & $\$ 115,321.48(\$ 60,637.07-\$ 170,005.89)$ & $\$ 226,523.28(\$ 167,948.18-\$ 285,098.38)$ & $<0.01$ \\
\hline
\end{tabular}

Boldface type indicates statistical significance.

with those reported in the literature and included postoperative bleeding (3.8\%), infection (3.8\%), and CSF leak $(0.9 \%)$.

\section{Discussion}

The surgical partnership model provided benefit to all parties involved. Most importantly, patient access to surgery was increased and surgery was provided by an experienced team performing a high volume of complex epilepsy surgery procedures. Partnering epileptologists benefited because they were actively involved in decision making and retained their patients postoperatively. Referring institutions benefited from increased revenue associated with performing noninvasive preoperative workup. Our institution benefited from the experience and revenue associated with an increase in the volume of complex cases. During the post-MOU time frame, the overall case volume and monthly charges increased significantly; monthly revenue and contribution margin also increased, although not significantly.

Referrals of complex cases from epilepsy centers with limited surgical capabilities to centers with high surgical volumes makes sense, intuitively, but execution may be problematic. This study described outcomes associated with a formalized partnership between centers. Less than $1 \%$ of patients with intractable epilepsy are referred for surgery. ${ }^{3}$ The results of our investigation demonstrated that our institutional partnerships provided an increase in surgically complex cases, as there was an increase in patients undergoing invasive studies and staged procedures to localize and treat the ictal-onset zone. Our partners were capable of performing noninvasive presurgical workups but were unable to consistently provide epilepsy surgery because of staff limitations. The patients also benefit because they were treated at a high-volume, experienced center, which has been shown to improve patient outcomes. . $^{10,13}$

The underutilization of epilepsy surgery is multifactorial. Despite high efficacy and low complication rates, only a small subset of epilepsy surgical candidates will undergo surgery, and often in a delayed manner. ${ }^{17}$ Poor referral patterns are likely not the only reason why appropriate candidates are not receiving surgical treatment. For example, a study showed that some patients simply decline surgery due to costs or fear. ${ }^{1}$ Indeed, a study performed at a tertiary epilepsy center in the United Kingdom showed that $32 \%$ of patients offered surgery declined to proceed, ${ }^{6}$ which may indicate that some appropriate candidates are not undergoing surgery because of personal preferences and preconceived notions regarding surgery. Another reason might be poor communication and relationships between community neurologists and surgical epilepsy centers. A survey of neurologists who treated patients with refractory epilepsy showed that approximately $50 \%$ of them were unsatisfied with the communication from epilepsy centers. ${ }^{7}$ To prevent that problem, we incorporated the referring epileptologists into our care; they presented their own cases in our epilepsy conferences, and patient care was transitioned back to the epileptologist after surgery. Thus our model of patients undergoing preoperative evaluation and postsurgical outpatient care at their original centers allowed for excellent continuity of care that fostered communication and involvement. The model streamlined the referral process, making it more efficient and effective.

Ultimately, the strategic partnerships benefited patients who were deemed appropriate candidates for surgery and might have had limited or no access to surgical treatment without these partnerships. A study of patients with temporal lobe epilepsy and hippocampal sclerosis showed that those who had longer duration of disease before undergoing resection also had poorer outcomes, implying that patients with medically refractory focal epilepsy should be considered for surgical treatment shortly after documented medication failure. ${ }^{9}$ Furthermore, patients who are seizure free after surgery have substantial increases in lifespan and quality of life in comparison to those receiving only medical treatment. ${ }^{2}$ Our goal was to provide a framework for other epilepsy centers to establish similar partnerships and to demonstrate that insurance and geographic location were not barriers to care.

\section{Conclusions}

This study describes a novel means to create strategic partnerships with nearby epilepsy centers with limited epilepsy surgery capabilities, increasing access to surgical care for patients with refractory epilepsy. Our method fosters effective communication and better relationships 
TABLE 4. Summary statistics of complex cases

\begin{tabular}{lc}
\hline \multicolumn{1}{c}{ Variable } & No. of Cases* \\
\hline Electrode type & \\
\hline SEEG & 25 \\
\hline SDG & 44 \\
\hline Therapy after invasive monitoring \\
\hline RNS & 21 \\
\hline VNS & 2 \\
\hline Resection & 39 \\
\hline Other & 8 \\
\hline
\end{tabular}

* Combined data for pre-MOU and post-MOU periods for $\mathrm{UCl}$ and partner institutions.

between centers, which may help overcome some referral barriers. Other centers may benefit from similar partnerships with nearby epilepsy centers, which would ultimately increase access to surgery and improve patient care.

\section{References}

1. Anderson CT, Noble E, Mani R, Lawler K, Pollard JR: Epilepsy surgery: factors that affect patient decision-making in choosing or deferring a procedure. Epilepsy Res Treat 2013:309284, 2013

2. Choi H, Sell RL, Lenert L, Muennig P, Goodman RR, Gilliam FG, et al: Epilepsy surgery for pharmacoresistant temporal lobe epilepsy: a decision analysis. JAMA 300:24972505, 2008

3. Engel J Jr: Why is there still doubt to cut it out? Epilepsy Curr 13:198-204, 2013

4. Engel J Jr, Wiebe S, French J, Sperling M, Williamson P, Spencer D, et al: Practice parameter: temporal lobe and localized neocortical resections for epilepsy. Epilepsia 44:741-751, 2003

5. Englot DJ, Ouyang D, Garcia PA, Barbaro NM, Chang EF: Epilepsy surgery trends in the United States, 1990-2008. Neurology 78:1200-1206, 2012

6. Fois C, Kovac S, Khalil A, Uzuner GT, Diehl B, Wehner T, et al: Predictors for being offered epilepsy surgery: 5-year experience of a tertiary referral centre. J Neurol Neurosurg Psychiatry 87:209-211, 2016

7. Hakimi AS, Spanaki MV, Schuh LA, Smith BJ, Schultz L: A survey of neurologists' views on epilepsy surgery and medically refractory epilepsy. Epilepsy Behav 13:96-101, 2008

8. Haneef Z, Stern J, Dewar S, Engel J Jr: Referral pattern for epilepsy surgery after evidence-based recommendations: a retrospective study. Neurology 75:699-704, 2010
9. Janszky J, Janszky I, Schulz R, Hoppe M, Behne F, Pannek HW, et al: Temporal lobe epilepsy with hippocampal sclerosis: predictors for long-term surgical outcome. Brain 128:395-404, 2005

10. Jette N, Wiebe S: Update on the surgical treatment of epilepsy. Curr Opin Neurol 26:201-207, 2013

11. Jobst BC, Cascino GD: Resective epilepsy surgery for drugresistant focal epilepsy: a review. JAMA 313:285-293, 2015

12. Kwan P, Brodie MJ: Early identification of refractory epilepsy. N Engl J Med 342:314-319, 2000

13. Labiner DM, Bagic AI, Herman ST, Fountain NB, Walczak TS, Gumnit RJ: Essential services, personnel, and facilities in specialized epilepsy centers-revised 2010 guidelines. Epilepsia 51:2322-2333, 2010

14. Pestana Knight EM, Schiltz NK, Bakaki PM, Koroukian SM, Lhatoo SD, Kaiboriboon K: Increasing utilization of pediatric epilepsy surgery in the United States between 1997 and 2009. Epilepsia 56:375-381, 2015

15. Roberts JI, Hrazdil C, Wiebe S, Sauro K, Vautour M, Wiebe $\mathrm{N}$, et al: Neurologists' knowledge of and attitudes toward epilepsy surgery: a national survey. Neurology 84:159-166, 2015

16. Rolston JD, Englot DJ, Knowlton RC, Chang EF: Rate and complications of adult epilepsy surgery in North America: analysis of multiple databases. Epilepsy Res 124:55-62, 2016

17. Vadera S, Griffith SD, Rosenbaum BP, Seicean A, Kshettry VR, Kelly ML, et al: National trends and in-hospital complication rates in more than 1600 hemispherectomies from 1988 to 2010: a Nationwide Inpatient Sample study. Neurosurgery 77:185-191, 2015

18. Wiebe S, Blume WT, Girvin JP, Eliasziw M: A randomized, controlled trial of surgery for temporal-lobe epilepsy. N Engl J Med 345:311-318, 2001

\section{Disclosures}

The authors report no conflict of interest concerning the materials or methods used in this study or the findings specified in this paper.

\section{Author Contributions}

Conception and design: Vadera, Mnatsakanyan, Sazgar, Lin, Hsu. Acquisition of data: Vadera, Chan. Analysis and interpretation of data: Vadera, Chan, Sen-Gupta, Hsu. Drafting the article: all authors. Critically revising the article: all authors. Reviewed submitted version of manuscript: Vadera, Chan, Mnatsakanyan, Sazgar, Lin, Hsu. Statistical analysis: Chan. Administrative/technical/material support: Vadera. Study supervision: Vadera, Hsu.

\section{Correspondence}

Sumeet Vadera: UC Irvine Medical Center, Orange, CA. svadera1@uci.edu. 